\title{
Correcting for respiratory motion in liver PET/MRI: preliminary evaluation of the utility of bellows and navigated hepatobiliary phase imaging
}

Thomas A. Hope ${ }^{1,2^{*}}$, Emily F. Verdin ${ }^{1}$, Emily K. Bergsland ${ }^{3}$, Michael A. Ohliger ${ }^{1,4}$, Carlos U. Corvera ${ }^{5}$ and Eric K. Nakakura ${ }^{5}$

* Correspondence:

thomas.hope@ucsf.edu

'Department of Radiology and Biomedical Imaging, University of

California, San Francisco, San Francisco, CA, USA

${ }^{2}$ Department of Radiology, San Francisco VA Medical Center, San Francisco, CA, USA

Full list of author information is available at the end of the article

\begin{abstract}
Background: The purpose of this study was to evaluate the utility of bellows-based respiratory compensation and navigated hepatobiliary phase imaging to correct for respiratory motion in the setting of dedicated liver PET/MRI.
\end{abstract}

Methods: Institutional review board approval and informed consent were obtained. Six patients with metastatic neuroendocrine tumor were imaged using Ga-68 DOTA-TOC PET/MRI. Whole body imaging and a dedicated 15-min liver PET acquisition was performed, in addition to navigated and breath-held hepatobiliary phase (HBP) MRI. Liver PET data was reconstructed three ways: the entire data set (liver PET), gated using respiratory bellows (RC-liver PET), and a non-gated data set reconstructed using the same amount of data used in the RC-liver PET (shortened liver PET). Liver lesions

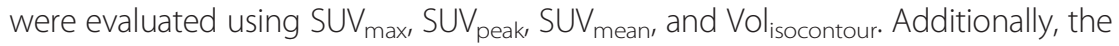
displacement of each lesion between the RC-liver PET images and the navigated and breath-held HBP images was calculated.

Results: Respiratory compensation resulted in a $43 \%$ increase in SUVs compared to ungated data (liver vs RC-liver PET SUV $\max 26.0$ vs $37.3, p<0.001$ ) and a $25 \%$ increase compared to a non-gated reconstruction using the same amount of data (RC-liver vs shortened liver PET SUV $\max _{2} 26.0$ vs $32.6, p<0.001$ ). Lesion displacement was minimized using navigated HBP MRI $(1.3 \pm 1.0 \mathrm{~mm})$ compared to breath-held HBP MRI (23.3 $\pm 1.0 \mathrm{~mm})$.

Conclusions: Respiratory bellows can provide accurate respiratory compensation when imaging liver lesions using PET/MRI, and results in increased SUVs due to a combination of increased image noise and reduced respiratory blurring.

Additionally, navigated HBP MRI accurately aligns with respiratory compensated PET data.

\section{Background}

The introduction of simultaneous PET/MRI promises to combine the soft tissue resolution associated with MRI and the high sensitivity and specificity of PET imaging. One of the difficult aspects of simultaneous imaging is how to appropriately leverage prolonged single bed position MR imaging into a whole body PET protocol [1-3]. This issue is fairly straightforward for brain and pelvis applications as there is minimal motion associated with the imaging and boils down to MR sequence selection. For 
chest and abdominal applications, such as liver imaging, respiratory motion can create issues for both MRI and PET imaging $[4,5]$.

Unlike conventional PET/CT acquisitions, patients perform numerous breath-holds throughout dedicated liver MR imaging markedly displacing the anatomy throughout the acquisition (Fig. 1). This can result in motion artifacts and errors in quantification. Additionally, localization of PET data to specific lesions seen on MRI can be difficult due to the displacement. MR sequences are often acquired during inspiratory breathholds, while PET data is predominantly acquired during end expiration.

Therefore, there is a need to develop respiratory compensation techniques to remove respiratory artifact from the PET data, as well as techniques to acquire MRI images that accurately localize with the PET data. In this study, we imaged six patients with metastatic neuroendocrine tumor to the liver using Ga-68 DOTA-TOC PET/MRI. We evaluated the use of respiratory bellows-compensated PET and navigated hepatobiliary phase imaging in order to address the issues with respiratory motion in liver imaging.

\section{Methods}

This study was approved by the local institutional review board, the UCSF Committee for Human Research and informed consent was obtained on all patients. Six patients were imaged between October 2014 and April 2015 with an average age of 65.5 years (three men and three women). Three of the patients were previously reported [6].

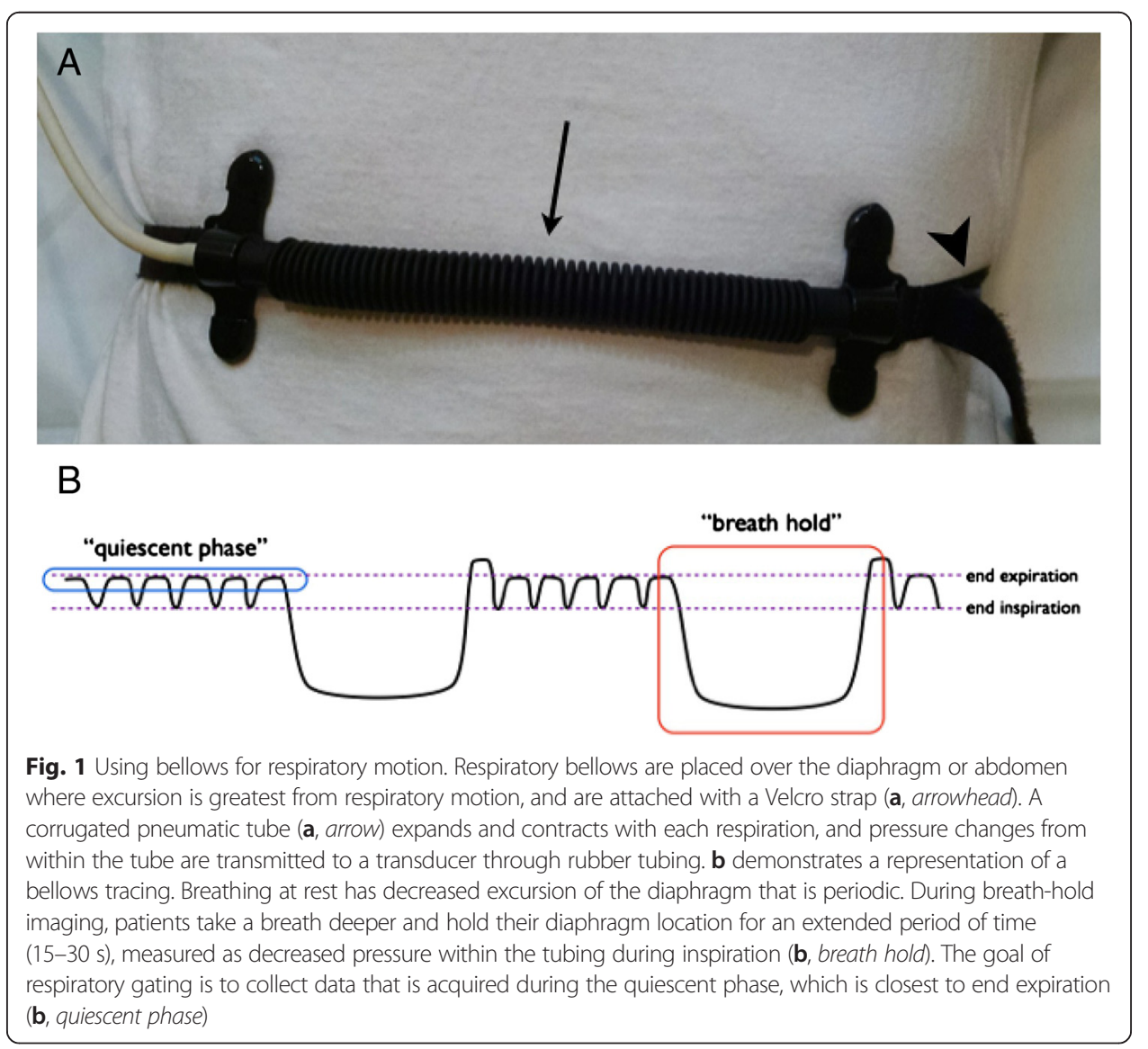




\section{Imaging protocol}

Patients were imaged on a 3.0T GE Signa PET/MRI (GE Healthcare, Waukesha, WI). Each patient was injected with $5.0 \pm 0.6 \mathrm{mCi}$ of Ga-68 DOTA-TOC. PET/MRI imaging began an average of $117 \pm 7 \mathrm{~min}$ after injection. The acquisition was delayed due to a preceding PET/CT each patient had; this delay results in a decreased amount of PET activity available for the PET/MRI acquisition. Each exam began with a six-bed position whole body PET/MRI with 2:15 s of acquisition time for PET at each bed position. During the whole body PET acquisition, a single breath-hold was performed for the T1-spoiled gradient echo acquisition. Subsequently, a liver specific bed position was acquired. During this bed position, precontrast (breath-held), dynamic postcontrast spoiled gradient echo imaging (breath-held), axial (breath-held) and coronal (free-breathing) single shot fast spin echo T2-weighted imaging, axial diffusion weighted imaging (free-breathing), and finally an axial navigated (free-breathing) and a breath-held hepatobiliary phase (HBP) spoiled gradient echo imaging were performed with the following parameters: slice thickness $=4 \mathrm{~mm}$, flip angle $=35^{\circ}$, matrix size $=320 \times 224, \mathrm{TE} / \mathrm{TR}=2.0 / 5.5, \mathrm{NEX}=0.7$. Patients were injected with $10 \mathrm{~mL}$ of gadoxetate disodium (Eovist, Bayer Healthcare), and HBP imaging was performed 10-20 min after injection when the hepatic parenchyma was enhanced due to hepatobiliary excretion. For breath-held imaging, patients were asked to take in a breath and hold their breath. Navigation was performed using a pencil navigator over the diaphragm, and MR data was acquired during end expiration [7]. In short, a pencil navigator acquires a linear excitation through the dome of the liver continuously throughout the acquisition and is used to track to location of the diaphragm. A high flip angle was used due to the increased intrinsic T1 contrast during the HBP [8].

\section{PET reconstruction}

All of the following PET data sets were reconstructed using a time-of-flight reconstruction with OSEM using two iterations and 28 subsets, and a matrix size of $256 \times 256$. The PET transaxial and z-axis field of view are 600 and $250 \mathrm{~mm}$, resulting in a voxel size of $2.3 \times 2.3 \mathrm{~mm}$. Axial slices were reconstructed at $2.78 \mathrm{~mm}$ in thickness. Attenuation correction was performed using a two-echo Dixon fat-water separation algorithm for the body while the lung was segmented using a region growing algorithm, which is standard on the scanner [1]. Attenuation correction MR data was acquired during shallow breathing. Using respiratory triggers obtained from bellows, the following PET data reconstructions were obtained of the liver:

1) WB PET: non-gated PET from the whole body PET/MRI, a 2:15 s acquisition.

2) Liver PET: non-gated PET from the 15-min liver bed position. Dedicated liver PET imaging began an average of $31 \pm 7$ min after the beginning of the whole body acquisition.

3) RC-liver PET: respiratory compensated (Qstatic, GE Healthcare, Waukesha, WI) PET data from the 15-min liver bed position [9]. A respiratory bellows is a pressure sensitive band that surrounds a patient's abdomen; as a patient breathes, the band expands and contracts to create respiratory waveforms (Fig. 1) [10]. Using the waveform from the bellows, respiratory triggers are created to denote the beginning of each inspiration. Fifty percent of the 
respiratory period from accepted breath-holds was included in the final reconstruction in order to use data only during end expiration when diaphragm motion is minimized. The $50 \%$ window began at a $30 \%$ delay from the bellows trigger so encompassed data between 30 and $80 \%$ of the respiratory cycle relative to the bellows trigger.

Any respiration that is longer than $10 \mathrm{~s}$ or less than $2 \mathrm{~s}$ in duration was excluded. On average, there were 91 accepted triggers and 25 rejected triggers from the total PET acquisition. On average $3.3 \pm 1.3 \mathrm{~min}$ of PET data was used to reconstruct the RC-liver PET data sets.

4) Shortened liver PET: because changes in SUV can be due to both changes in noise and respiratory compensation, we created a "shorted" PET reconstruction that did not incorporate respiratory compensation, but used the same amount of PET data as the RC-liver PET reconstruction. For this reason, shortened non-gated PET data from the 15-min liver bed was reconstructed utilizing the same amount of PET data used to make the RC-liver PET data set. Respiratory trigger data was evaluated to only include PET data acquired during regular respirations in order to exclude long breath-holds in this data set (Fig. 2).

\section{Qualitative evaluation of motion artifact}

The four PET reconstructions were graded qualitatively for the presence of motion artifact. Qualitative characterization was performed on coronal reformats. Motion was graded on a linear 1-5 scale (1: no motion artifact, 2: mild blurring without ghosting,

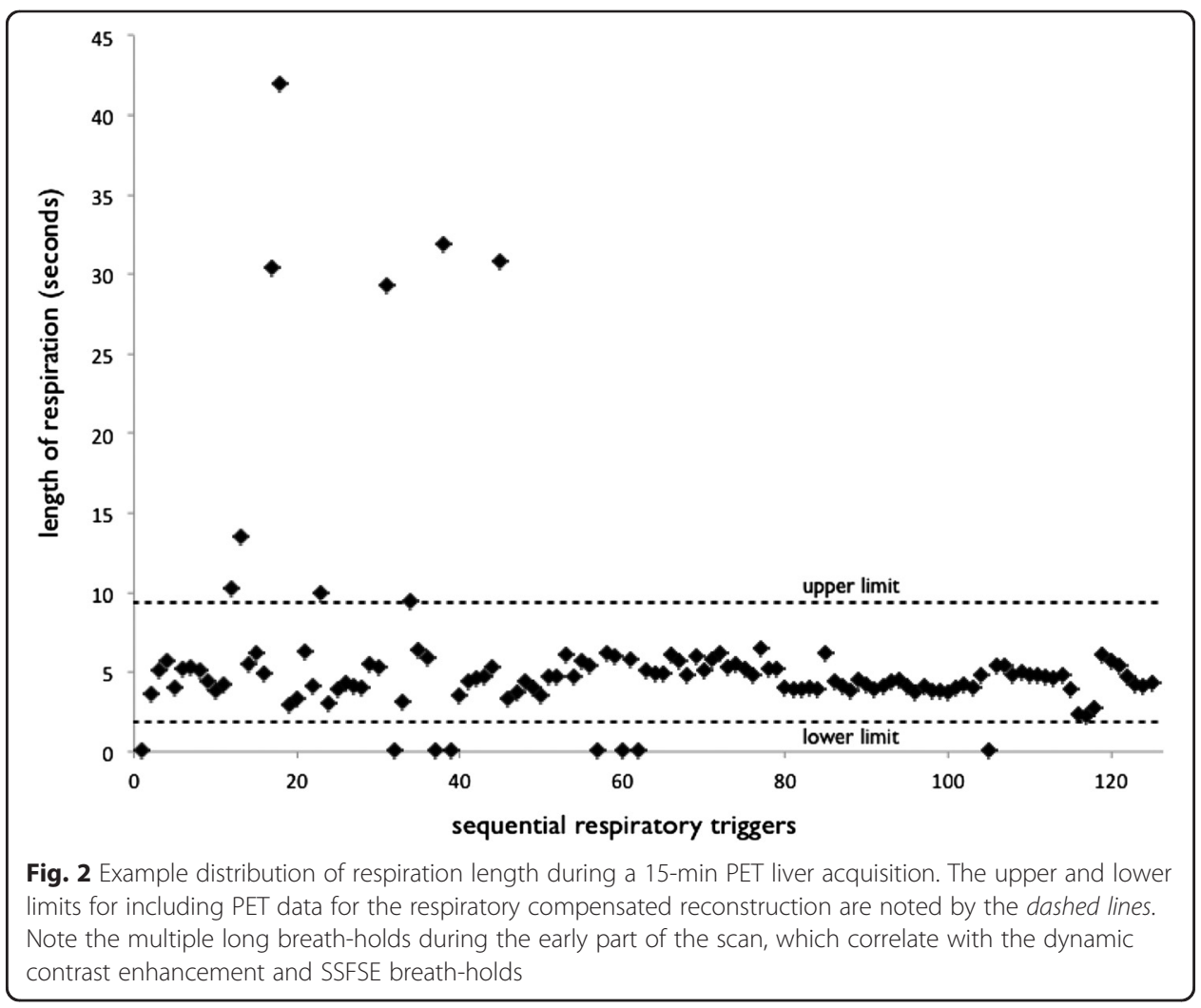


3: significant blurring without ghosting, 4: blurring and minimal ghosting, 5: marked blurring and ghosting lesions).

\section{Effect of respiratory gating on SUV measurements}

Hepatic lesions between 1 and $5 \mathrm{~cm}$ in diameter that are distinct from adjacent lesions were included for analysis. Additionally, lesions that were not able to be segmented using a threshold due to low uptake compared to the background liver or difficulty segmenting from adjacent lesions were excluded. For each lesion, the $\mathrm{SUV}_{\max }, \mathrm{SUV}_{\text {mean }}$, and $\mathrm{SUV}_{\text {peak }}$ were calculated. $\mathrm{SUV}_{\text {peak }}$ is measured as the average value in a $1-\mathrm{cm}^{3}$ sphere centered around the maximum voxel within an ROI. SUV mean and $\mathrm{Vol}_{\text {isocontour }}$ were calculated using a threshold-based technique with an isocontour line defined by $42 \%$ of the maximum activity within the ROI. All measurements were performed using an Advantage Workstation 5.0 (GE Healthcare, Waukesha WI).

\section{Lesion excursion evaluation}

Hepatic lesions between 1 and $5 \mathrm{~cm}$ in diameter that are distinct from adjacent lesions were included for analysis. The center of each lesion on coronal MIP PET images was determined using Qstatic reconstruction, and the z-axis location was recorded. Similarly, the z-axis location of the center of each lesion was also recorded on coronal reconstructions of breath-held and navigated hepatobiliary imaging. The difference in location between the MR and PET acquisitions was then calculated.

\section{Noise evaluation}

Noise was evaluated as previously described for liver parenchyma. A region of interest (ROI) was placed manually over liver parenchyma in the left and right hepatic lobes being careful not to include any focal lesions. Additionally, an ROI was placed anterior the patient in a region of uniform background activity. Noise is defined as the standard deviation of signal intensity of the liver divided by the average signal intensity of the liver within each ROI [11].

\section{Statistical analysis}

All scale variables are presented as means and standard deviations. Comparison of means was performed using a paired Student's $t$ test. The Wilcoxon signed-rank test was used to compare qualitative scoring of motion artifact. A $p$ value less than 0.05 was considered significant. All analyses were performed using R [12].

\section{Results}

All imaged patients had DOTA-TOC avid hepatic lesions. Evaluation of breath-hold length demonstrated that there were multiple long breath-holds throughout the liver acquisition (Fig. 2), which correlates with breath-hold MRI sequences such as the dynamic contrast enhancement and single-shot fast spin-echo sequences (SSFSE). Qualitatively, the hepatic lesions demonstrated respiratory blurring and ghosting that was removed when applying bellows-based respiratory compensation (Fig. 3). Qualitative motion artifact was significantly less in the liver PET compared to the three other PET reconstructions (Table $1, p$ values ranging from $<0.001$ to 0.03 ). 


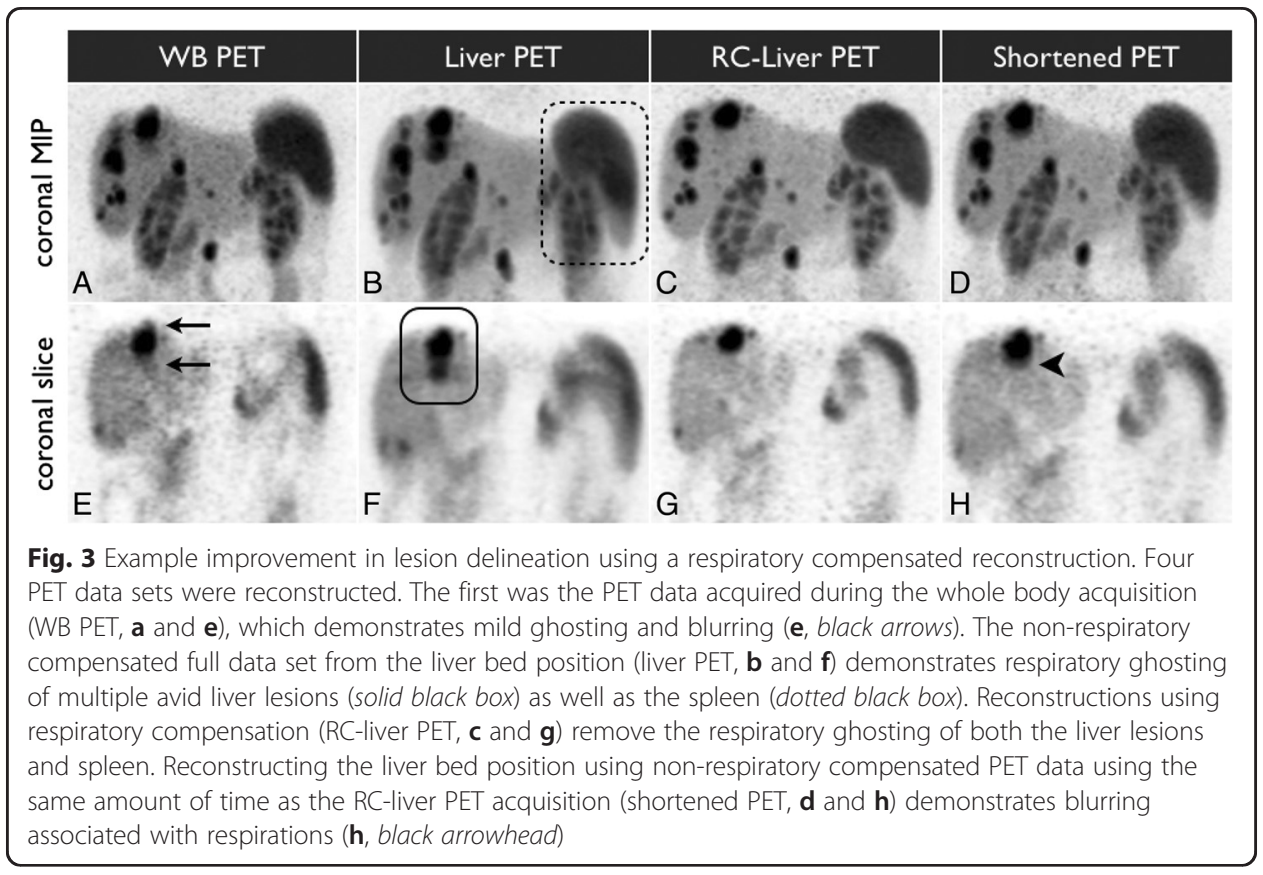

\section{Effect of respiratory gating on SUV measurements}

Twelve individual lesions were included for analysis (Fig. 4, Table 1). RC-liver PET had a $\mathrm{SUV}_{\text {max }}$ and $\mathrm{SUV}_{\text {peak }}$ greater than the liver PET (43 and $38 \%$ greater, both $p$ values $<0.001)$ and the shortened liver PET (14 and $13 \%$ greater, $p<0.001$ and $p=0.008)$. The shortened liver PET had a $\mathrm{SUV}_{\text {max }}$ and $\mathrm{SUV}_{\text {peak }}$ greater than the liver PET (25 and $23 \%$ greater, both $p$ values $<0.001$ ).

Respiratory compensation (RC-liver PET) resulted in a significant decrease in $\mathrm{Vol}_{\text {isocontour }}$ compared to the liver PET $\left(3.7 \pm 3.6 \mathrm{~cm}^{3}\right.$ vs $5.4 \pm 4.5,31 \%$ decrease; $\left.p=0.001\right)$. There was significantly larger $\mathrm{Vol}_{\text {isocontour }}$ with the liver PET compared to the WB PET likely due to the numerous breath-holds performed during the dedicated liver imaging (25\% greater, $p=0.009)$.

\section{Lesion excursion}

Sixteen individual lesions were included in the lesion excursion evaluation. Navigated HBP MRI resulted in accurate registration to the RC-liver PET data, compared to breath-held MRI where there was noted misregistration (Fig. 5). The average distance

Table 1 Average SUV max, SUV mean, SUV peak, Vol isocontour, and artifact score for each of the four PET reconstructions

\begin{tabular}{|c|c|c|c|c|}
\hline & WB PET & Liver PET & RC-liver PET & Shortened PET \\
\hline $\mathrm{SUV}_{\max }$ & $33.0 \pm 9.8^{*}$ & $26.0 \pm 8.8^{*}$ & $37.3 \pm 13.3$ & $32.6 \pm 10.5^{*}$ \\
\hline$S_{\text {SUean }}$ & $20.2 \pm 6.3^{*}$ & $15.8 \pm 5.2^{*}$ & $23.4 \pm 8.7$ & $20.4 \pm 6.5^{*}$ \\
\hline SUV & $23.5 \pm 8.5^{*}$ & $20.0 \pm 7.5^{*}$ & $27.6 \pm 12.0$ & $24.5 \pm 9.4^{*}$ \\
\hline$V_{\text {ol isocontour }}\left(\mathrm{cm}^{3}\right)$ & $4.3 \pm 4.5$ & $5.4 \pm 4.5^{*}$ & $3.7 \pm 3.6$ & $4.4 \pm 4.3^{*}$ \\
\hline Artifact score & $3.2 \pm 0.8^{*}$ & $4.5 \pm 0.5^{*}$ & $1.2 \pm 0.4$ & $2.7 \pm 1.2^{*}$ \\
\hline
\end{tabular}

${ }^{*} p$ value $<0.05$ compared to RC-liver PET 


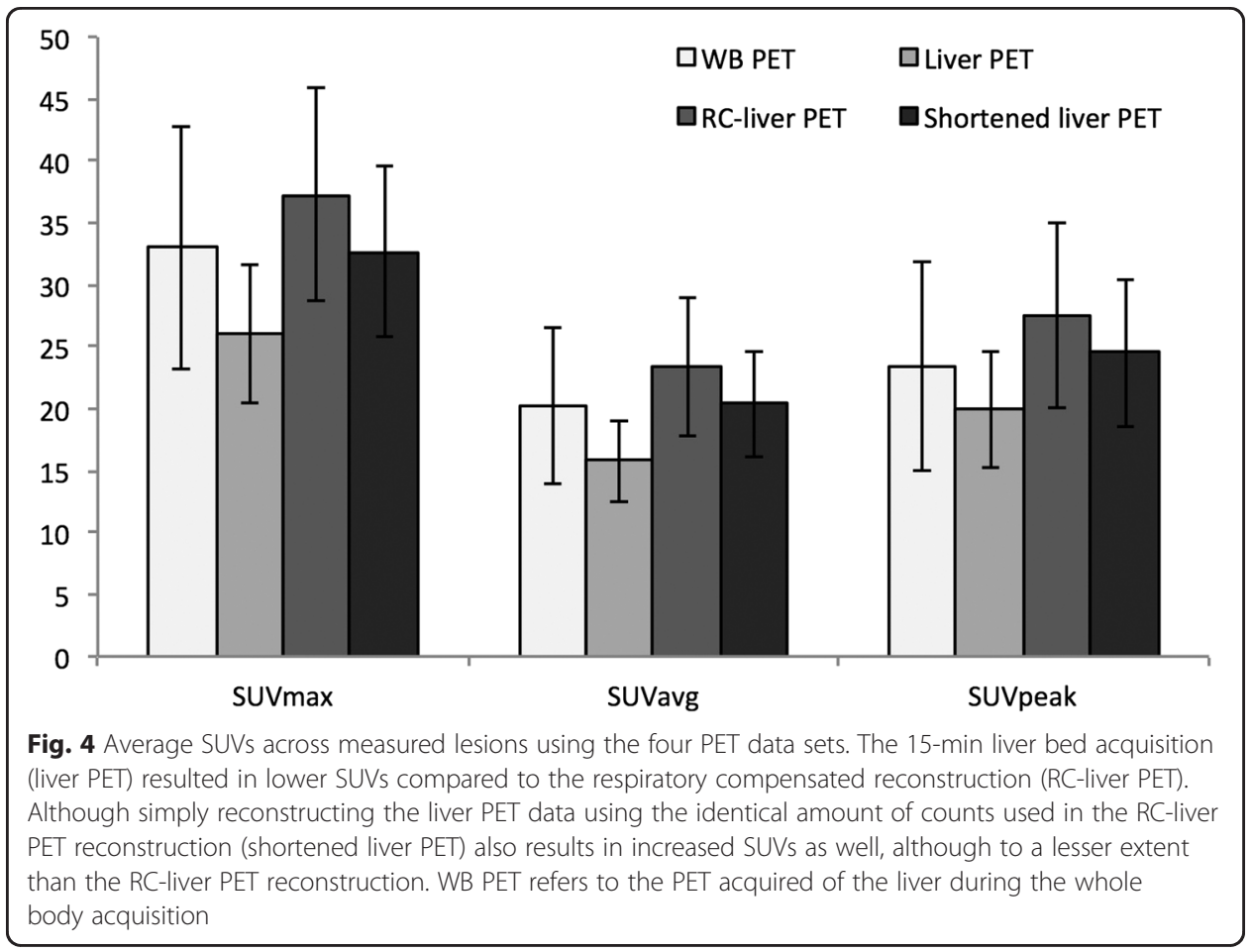

between the center of the lesion on RC-liver PET and on breath-held MRI was $23.3 \pm 8.1 \mathrm{~mm}(p<0.001)$, while the average distance between the center of the lesions on RC-liver PET and on navigated MRI was $1.3 \pm 1.0 \mathrm{~mm}(p=0.3)$. Fusion using navigated HBP imaging and respiratory compensated PET data resulted in accurate fusion (Fig. 6).

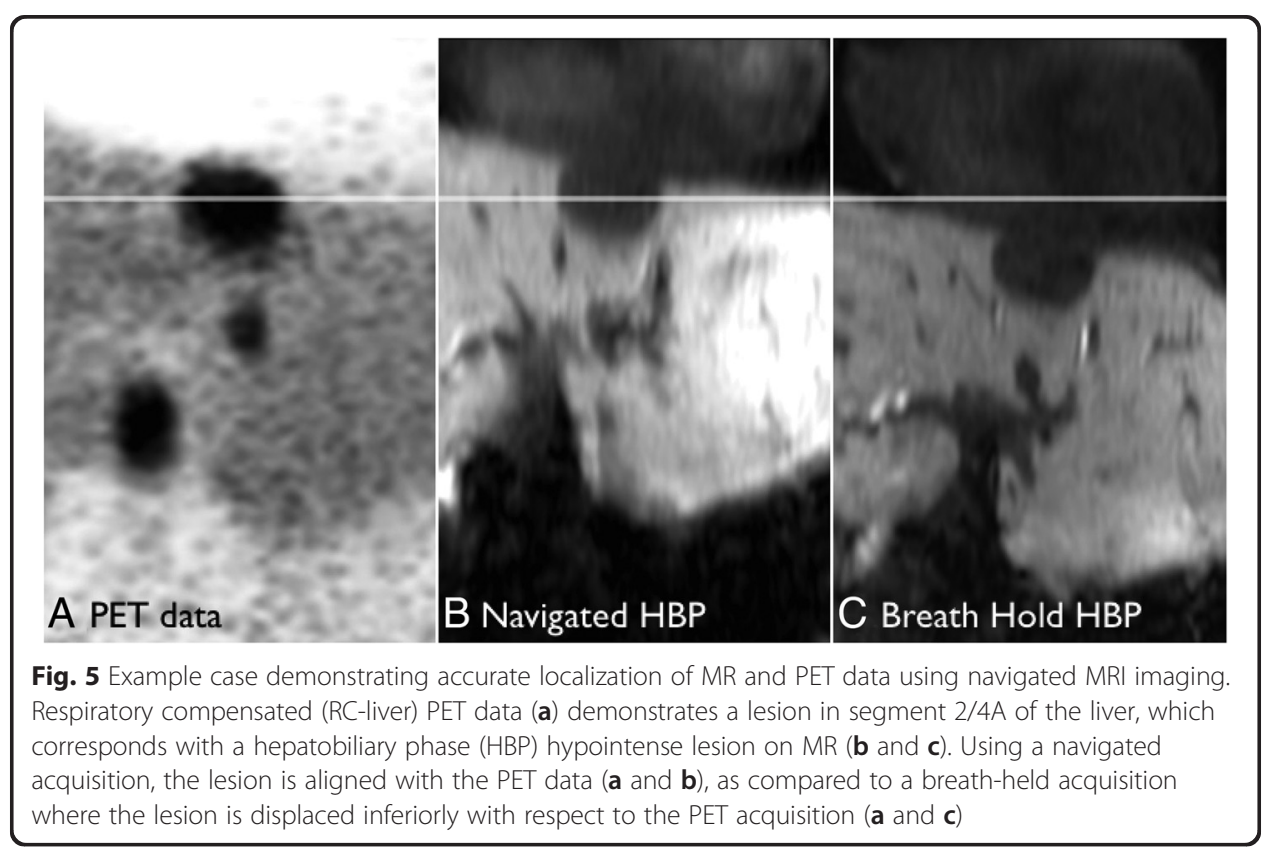




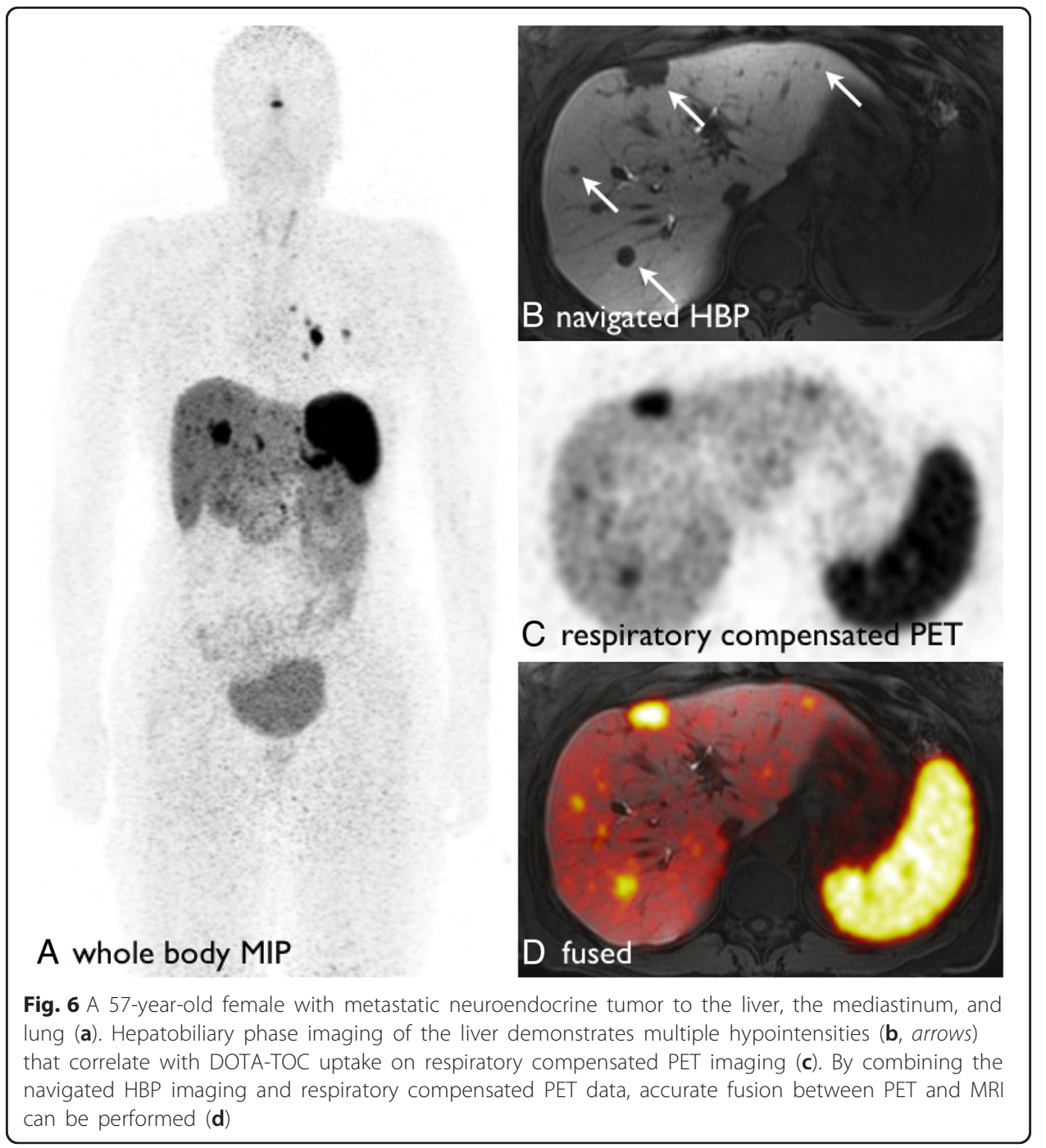

\section{Noise evaluation}

The average noise measurements in the liver for the WB PET, liver PET, RC-liver PET, and shortened liver PET were $0.23,0.13,0.23$, and 0.21 , respectively. The noise in the liver PET was significantly lower than in the RC-liver PET and shortened liver PET ( $p=0.016$ and 0.019). There was no significant difference in noise between the RC-liver PET and shortened liver PET $(p=0.22)$.

\section{Discussion}

We have demonstrated that the incorporation of respiratory compensation into PET reconstruction can aid in removing respiratory artifact in PET/MRI. Using bellows is a convenient way to incorporate respiratory gating without affecting MRI imaging that is already included in clinically available scanners. Of note, the measured SUVs were higher using respiratory compensation (RC-liver PET) due to increased image noise and removal of motion artifact compared to the full acquisition (liver PET), and when corrected for scan time (shortened liver PET), respiratory compensation resulted in a 
slight increase in SUVs. Additionally, using a navigated hepatobiliary phase resulted in improved alignment of PET and MRI data allowing for accurate fusion.

There are two reasons that SUV values would be different when incorporating respiratory compensation. First, respiratory compensation decreases the amount of PET data used in reconstruction resulting in more noise in the data set. Previous work using FDG has demonstrated that $\mathrm{SUV}_{\max }$ calculations are sensitive to noise and values decrease with longer scan times as image noise decreases [13]. Separate from the issue of noise, the removal of respiratory blurring will increase measured SUVs [14, 15]. In order to separate these two effects, we reconstructed a non-respiratory compensated data set (shortened liver PET) that used the identical amount of data as in the respiratory compensated reconstruction (RC-liver PET) in order to create datasets with the same amount of noise. We demonstrated that the effect of increased noise and removal of respiratory blurring both resulted in increased SUVs as expected. Previous work has also shown that respiratory gating in the setting of PET/MRI results in increased SUVs but did not take into account changes in image noise [16].

Similar approaches to performing respiratory compensation have been used in abdominal PET/CT using sensor belts around the abdomen [17, 18]. Other techniques have used a positioning monitoring system that involves an infrared camera, although this may not be translatable to the PET/MRI setting due to the narrow bore size, anterior coils, and the magnetic field [19]. It is also possible to use MRI navigators to provide gating information in order to reconstruct respiratory gated PET data [16, 20, 21]. This approach is limited as it requires MRI data to be continuously acquired throughout the PET acquisition, limiting what MR data you can acquire. Other approaches use MR data to create deformation maps that allow one to register PET data acquired at different time points in the respiratory cycle to create a respiratory gated data set without removing motion corrupted data [22]. Finally, one can use the PET data itself to perform motion correction although these approaches are limited due to noise [23, 24]. One recent interesting approach is to use a training dataset to inform how respiratory motion can be gated using list mode PET data itself, although this requires a short acquisition after the completion of the study [5]. Overall, we believe the use of bellowsbased respiratory gating provides a simple robust way to gate PET/MRI data without affecting MR imaging.

We report the first use of diaphragm-navigated HBP imaging for PET/MRI and demonstrated that gated MR acquisition during end expiration results in an MR image that accurately fuses with respiratory compensated PET data. Other approaches to address this issue could be to perform a radial free-breathing acquisition as it results in an image that is an average throughout free-breathing similar to PET data, although this has not been evaluated in the setting of PET/MRI [25].

One important point is that image noise greatly influences SUVs. Typically PET/ $\mathrm{CT}$ is done with a standard amount of time at each bed position. With PET/MRI, due to the need to perform numerous MRI sequences [26], one may decide to increase the amount of time acquiring data at a single bed position. Although the increased frame time can increase lesion detection and improve image quality, the differences in frame time can result in nearly 30 \% differences in SUVs. Therefore, when evaluating for changes in SUV, it is critical to choose PET data sets with similar frame times. 
There are a number of limitations associated with this study. First is the limited number of patients and lesions imaged, which will require further work to validate the findings in this study. Second, the same attenuation correction map was used for all PET data reconstructions, and so differences in SUV values may be due to misregistration between PET and MRAC caused by respiratory motion [27]. Third, the increased delay time for PET/ MRI compared to routine clinical imaging time points of 55-70 min results in decreased activity resulting in more noise than may be present at shorter imaging delays.

\title{
Conclusions
}

In conclusion, we have demonstrated the utility of respiratory bellows for providing respiratory compensation when imaging liver lesions using PET/MRI using existing software available on clinical PET/MR systems. Respiratory compensation results in increased SUVs both due to increased image noise and reduced respiratory blurring. Finally, navigated HBP MRI provides high quality images that accurate align with respiratory compensated PET data.

\section{Ethical approval}

All procedures performed were in accordance with the ethical standards of the local institutional research committee.

\section{Informed consent}

Informed consent was obtained from all individual participants included in the study.

Competing interests

Thomas A. Hope has received speaker honoraria and grant support from GE Healthcare. Emily Verdin, Emily K. Bergsland, Michael A. Ohliger, Carlos U. Corvera MD, and Eric K. Nakakura declare that they have no competing interests.

\section{Authors' contributions}

$\mathrm{TH}$ participated in the design and method development of the study, measured the lesion SUVS, performed the statistical analysis, and prepared the manuscript. EV reconstructed the simulated PET data sets, consented patients, coordinated patient visits, and revised the manuscript. EB participated in developing the study design, aided in patient recruitment and helped revise the manuscript. $\mathrm{MO}$ participated in the design and method development of the study and helped revise the manuscript. CC and EN participated in the design and method development of the study, recruited patients, and helped to draft the manuscript and revised it critically. All authors read and approved the final manuscript.

\section{Acknowledgements}

We would like to thank Timothy Deller and Floris Jansen for aiding in PET reconstruction and manuscript review.

\author{
Author details \\ 'Department of Radiology and Biomedical Imaging, University of California, San Francisco, San Francisco, CA, USA. \\ 2Department of Radiology, San Francisco VA Medical Center, San Francisco, CA, USA. ${ }^{3}$ Division of Hematology/ \\ Oncology, Department of Medicine, University of California, San Francisco, San Francisco, CA, USA. ${ }^{4}$ Department of \\ Radiology, San Francisco General Hospital, San Francisco, CA, USA. ${ }^{5}$ Division of Surgical Oncology, Department of \\ Surgery, University of California, San Francisco, San Francisco, CA, USA.
}

Received: 22 June 2015 Accepted: 11 September 2015

Published online: 18 September 2015

References

1. Wollenweber SD, Ambwani S. Comparison of 4-class and continuous fat/water methods for whole-body, MRbased PET attenuation correction. IEEE Trans Nucl Sci. 2013;60(5):3391-98.

2. Barnwell J, Raptis CA, McConathy JE, Laforest R, Siegel BA, Woodard PK, et al. Beyond whole-body imaging: advanced imaging techniques of PET/MRI. Clin Nucl Med. 2014;40(2):e88-95.

3. Martinez-Möller A, Eiber M, Nekolla SG, Souvatzoglou M, Drzezga A, Ziegler S, et al. Workflow and scan protocol considerations for integrated whole-body PET/MRI in oncology. J Nucl Med. 2012;53(9):1415-26.

4. Nehmeh SA, Erdi YE. Respiratory motion in positron emission tomography/computed tomography: a review. Semin Nucl Med. 2008;38:167-76. 
5. Manber R, Thielemans K, Hutton B, Barnes A, Ourselin S, Arridge S, et al. Practical PET respiratory motion correction in clinical PET/MR. J Nucl Med. 2015;56(6):890-6.

6. Hope TA, Pampaloni MH, Nakakura E, VanBrocklin H, Slater J, Jivan S, et al. Simultaneous (68)Ga-DOTA-TOC PET/MRI with gadoxetate disodium in patients with neuroendocrine tumor. Abdom Imaging. 2015;40(6):1432-40.

7. Nagle SK, Busse RF, Brau AC, Brittain JH, Frydrychowicz A, Iwadate Y, et al. High resolution navigated three-dimensional $\mathrm{T}_{1}$-weighted hepatobiliary MRI using gadoxetic acid optimized for 1.5 Tesla. J Magn Reson Imaging. 2012;36:890-9.

8. Bashir MR, Husarik DB, Ziemlewicz TJ, Gupta RT, Boll DT, Merkle EM. Liver MRI in the hepatocyte phase with gadolinium-EOB-DTPA: does increasing the flip angle improve conspicuity and detection rate of hypointense lesions? J Magn Reson Imaging. 2012;35:611-6.

9. Liu C, Alessio A, Pierce L, Thielemans K, Wollenweber S, Ganin A, et al. Quiescent period respiratory gating for PET/CT. Med Phys. 2010;37:5037.

10. François CJ, Lum DP, Johnson KM, Landgraf BR, Bley TA, Reeder SB, et al. Renal arteries: isotropic, high-spatialresolution, unenhanced MR angiography with three-dimensional radial phase contrast. Radiology. 2011:258:254-60.

11. Jakoby BW, Bercier Y, Conti M, Casey ME, Bendriem B, Townsend DW. Physical and clinical performance of the mCT time-of-flight PET/CT scanner. Phys Med Biol. 2011:56:2375-89.

12. R Core Team. R: A language and environment for statistical computing [Internet]. Vienna, Austria; 2013 Jan. Available from: http://www.R-project.org/.

13. Lodge MA, Chaudhry MA, Wahl RL. Noise considerations for PET quantification using maximum and peak standardized uptake value. J Nucl Med. 2012;53:1041-7.

14. Li G, Schmidtlein CR, Burger IA, Ridge CA, Solomon SB, Humm JL. Assessing and accounting for the impact of respiratory motion on FDG uptake and viable volume for liver lesions in free-breathing PET using respirationsuspended PET images as reference. Med Phys. 2014;41:091905.

15. Liu C, Pierce LA, Alessio AM, Kinahan PE. The impact of respiratory motion on tumor quantification and delineation in static PET/CT imaging. Phys Med Biol. 2009;54:7345-62.

16. Fürst S, Grimm R, Hong I, Souvatzoglou M, Casey ME, Schwaiger M, et al. Motion correction strategies for integrated PET/MR. J Nucl Med. 2015:56:261-9.

17. Van Der Gucht A, Serrano B, Hugonnet F, Paulmier B, Garnier N, Faraggi M. Impact of a new respiratory amplitudebased gating technique in evaluation of upper abdominal PET lesions. Eur J Radiol. 2014;83:509-15.

18. Daouk J, Fin L, Bailly P, Meyer ME. Respiratory-gated positron emission tomography and breath-hold computed tomography coupling to reduce the influence of respiratory motion: methodology and feasibility. Acta Radiol. 2009:50:144-55.

19. Wang S, Bowen SR, Chaovalitwongse WA, Sandison GA, Grabowski TJ, Kinahan PE. Respiratory trace feature analysis for the prediction of respiratory-gated PET quantification. Phys Med Biol. 2014;59:1027-45.

20. Grimm R, Fürst S, Souvatzoglou M, Forman C, Hutter J, Dregely I, et al. Self-gated MRI motion modeling for respiratory motion compensation in integrated PET/MRI. Med Image Anal. 2015:19:110-20.

21. Grimm R, Fürst S, Dregely I, Forman C, Hutter JM, Ziegler SI, et al. Self-gated radial MRI for respiratory motion compensation on hybrid PET/MR systems. Med Image Comput Comput Assist Interv. 2013:16:17-24.

22. Fayad H, Schmidt H, Würslin C, Visvikis D. Reconstruction incorporated respiratory motion correction in clinical simultaneous PET/MR imaging for oncology applications. J Nucl Med. 2015;56(6):884-9.

23. Catana C. Motion correction options in PET/MRI. Semin Nucl Med. 2015;45:212-23.

24. Dawood M, Buther F, Jiang X, Schafers KP. Respiratory motion correction in 3-D PET data with advanced optical flow algorithms. IEEE Trans Med Imaging. 2008:27:1164-75.

25. Reiner CS, Neville AM, Nazeer HK, Breault S, Dale BM, Merkle EM, et al. Contrast-enhanced free-breathing 3D T1-weighted gradient-echo sequence for hepatobiliary MRI in patients with breath-holding difficulties. Eur Radiol. 2013;23:3087-93.

26. Zeimpekis KG, Barbosa F, Hüllner $M$, Voert ter $E$, Davison $H$, Veit-Haibach $P$, et al. Clinical evaluation of PET image quality as a function of acquisition time in a new TOF-PET/MRI compared to TOF-PET/CT-initial results. Mol Imaging Biol. 2015. [Epub ahead of print].

27. Keller SH, Holm S, Hansen AE, Sattler B, Andersen F, Klausen TL, et al. Image artifacts from MR-based attenuation correction in clinical, whole-body PET/MRI. MAGMA. 2013;26:173-81. 